\title{
Study on Early Evaluation of Earthquake Housing Loss in Yunnan Province Based on a Joint Mixed Modeling Approach
}

\author{
Mao Zhao, Li Li, Xiangxiang Guo \\ Trans-Asian Business School \\ Yunnan normal university \\ Kunming, China \\ 306155414@qq.com
}

\author{
赵茂, 李丽, 郭向向 \\ 昆明650092, 中国 \\ 306155414@qq.com
} \\ 云南师范大学泛亚商学院}

基于联合模型的云南地震房屋损失早期评估研究

\begin{abstract}
Scientific and reasonable earthquake natural disaster assessment has become an important reference for government materials distribution and emergency rescue. It is particularly important to estimate the damage situation and distribution of houses. As a high frequency area of earthquakes, Yunnan shows the characteristics of "small earthquake disaster, strong earthquake disaster". There are great differences and regional characteristics in the damage of houses caused by earthquake. This paper uses the joint mixed modeling approach to evaluate the different types of damage in housing in order to provide scientific basis and reference for the early emergency rescue deployment for the first time.
\end{abstract}

\section{Keywords-Earthquake; Loss of housing; Differences}

摘要一科学合理的地震自然灾害评估成为政府物资发放、 应急救援的一个重要参考, 房屋破坏情况及分布的预估显得 尤为重要。云南作为地震的高发区, 表现出 “小震致大灾, 中强震致巨灾” 的特点。地震引起的房屋损失破坏程度存在 巨大的差异性和区域性特征, 本文首次运用联合模型进行房 屋的不同类型损失评估，以期为早期应急救援部署提供一定 的科学的依据和参考。

关键词一地震, 房屋损失, 差异性

$$
\text { I. 引言 }
$$

地震发生后，政府及救援机构关心人员伤亡、房屋 破坏、道路破坏情况及分布，并根据信息作出科学有效 的救援部署和安排，为救灾物资发放、人员的调配部署 提供科学的依据, 避免盲目的施救。美国、日本、台湾 等都建立了相应的地震应急快速评估系统。比如: TELE 系统 ${ }^{[1]}$ （Taiwan Earthquake Loss Estimation System）结合 地震的震级、震中位置和震源深度能快速的计算建筑物 的破坏数量、死亡人数等。PAGER 系统 ${ }^{[2] ~(P r o m p t ~}$ Assessment of Global Earthquake for Response system) 结 合地震参数及 ShakeMap 进行早期预测, 同时结合经 验、倒塌率等进行不断修正。QLARM ${ }^{[3]}$ （Earthquake
Loss Assessment for Response and Mitigation）采用烈度进 行建筑物破坏损失程度评估。日本的 Phoenix 系统 ${ }^{[4]}$

(Phoenix Disaster Management System) 主要通过 Web 向公众提供必要的灾害信息。先进的经验值得借鉴和学 习, 但由于知识产权的自主性及预测地震系统的区域性 差异, 很难进行移植。王瑛等 ${ }^{[5]}$ (2005) 通过烈度损失

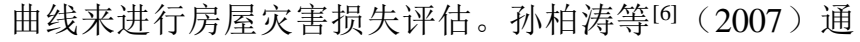
过回归模型构建了烈度区的人员伤亡、房屋损失的定量 关系。牛方曲等（2012） [7]基于县级尺度房屋结构、地 震烈度区划图等建立了中长期房屋损失系统。JinChen （2017） ${ }^{[8]}$ 基于甘肃省地震进行了抗震减灾能力评价。

通过文献的研判, 信息的越全面, 模型的预测越精 准。考虑地震的突发性及破坏性以及救援的及时性, 快 速的评估早期房屋损失为人员伤亡的救助、所需的帐 篷、食物等的提供一定的参考。一次地震发生, 将会出 现房屋破坏的差异, 表现出: 房屋毁坏、房屋破坏严 重、房屋破坏中等、房屋破坏轻微四种不同分类结果。 为此, 破坏程度的差异性也影响早期的救援安排的差异 性, 对于房屋损坏、房屋破坏严重更为关注。现有模型 很少结合分类数据进行联合实证分析。本文创新之处在 于：一是运用联合混合模型对四类不同房屋损失进行分 类别联合构建模型; 二是结合灾害发生初期, 很难获取 到的信息, 比如建筑物稠密度、建筑物质量等, 文章仅 选取能够时间相对快速监测到震级、震中深度、发震时 间、震中位置四个因素构造模型。三是地震的差异性, 云南属于丘陵和多民族地区，文化、建筑类型、居民生 活规律等有别于其他地区, 区域性研究更有针对性。为 此, 选取云南 1992-2010 年震级大于 4.7 (考虑到 4.7 级以下的震级影响不大的实际情况, 为此现有的灾评报 告主要统计震级 $\geqslant 4.7$ 的地震灾害) 的全样本数据进行研 究。期望为政府应急救援部署提供一定的科学的依据。 当然，联合混合模型的思想可推广应用。 
II. 模型及实证分析

\section{A. 模型设定}

分类数据经常发生在自然环境、生物、医学等方 面。地震自然灾害导致的房屋表现为: 房屋毁坏（记录 为 Destructive)、房屋破坏严重（记录为 Serious）、房 屋破坏中等（记录为 Medium）、房屋破坏轻微（记录 为 Minor) 四种不同分类结果。联合模型就是最新处理 多类数据的模型。该模型详见 Yan（2016）[9]一文，模 型简化如下:

$$
Y_{i 1}, Y_{i 2}, Y_{i 3}, Y_{i 4} \mid W, Y_{i 1}+Y_{i 2}+Y_{i 3}+Y_{i 4}=N_{i} \sim \text { 多项式 }\left(N_{i}, p_{i 1}, p_{i 2}, p_{i 3}, p_{i 4}\right)
$$

$Y_{i j}$ 表示 $\mathrm{i}$ 地区 $\mathrm{j}$ 类型的房屋损失, $p_{i 1}, p_{i 2}, p_{i 3}, p_{i 4}$ 表 示房屋毁坏、房屋破坏严重、房屋破坏中等、房屋破坏 轻微对应于多项式比例:

$$
p_{i j}=\frac{V_{i j} \exp \left(X_{i j}^{\prime} \beta_{j}\right)}{\sum_{l=1}^{4} V_{i l} \exp \left(X_{i l}^{\prime} \beta_{l}\right)} \quad j=1,2,3,4 ; i=1, \ldots, K
$$

\section{B. 指标说明}

由于中国统计年鉴起步较晚，早期的地震灾害数据 记录存在缺失。结合 1992-2010 年的云南完善灾评报告 进行分析云南地震对房屋损失的评估。数据来源于《云 南地震灾害损失评估及研究 1992-2010》。考虑到模型 用于早期的房屋损失评估，主要用于帐篷、救援物资的 配送参考，为此模型仅选取早期快速获取的指标如下：

1.地震的震级: 震级是地震能量释放的一个参数, 一般震级越大释放的能量越大, 房屋损失也会越大。地 震发生的首次记录, 记为 Magnitude。相比全球地震自 然灾害，在同等级震级下，云南地震伤亡更为严重。对 云南特大伤亡地震研究发现短时间多次发生同等级、或 更高级余震的现象明显，为此余震的引入显得尤为必 要。余震震级记为 Aftershocks, 计算方式为未发生余震 记录为 0 , 多次余震为其相应乘积。考虑首次地震与余 震的共同效应, 记为 Magnitude*Aftershocks 交互项作为 调节项; 2. 地震深度: 指地震中心位置与地面的距离, 多次地震实践表明深度越深，损失越小，记为 Depth； 3 . 地震发震的时间，Year 表示发震的年份，月份指标 $\cos (2 * \pi *$ month/12) (记录为 Cosmonth) 和 $\sin (2 * \pi *$ month $/ 12)$ （记录为 Sinmonth）作为周期性指 标来反映月份情况; 4. 地震位置: 考虑到地震位置的特 殊性, 一并考虑。结合中国经济发展的特色, 县域发展 一般优于村镇, 为此离县城越近的地方, 房屋建造的密 集度越大，地震造成的损失将会大; 离县城越远的地 方, 房屋建造的密集度越小，造成的损失越小。为此本 文采取震中位置 (经、纬度) 与灾害最近县城中心 （经、纬度）的欧式空间距离来反映地理位置的效应, 标记为 Distance。

\section{C. 实证分析}

经过实证如表 1，对于房屋毁坏（Destructive），地 震震级与余震越大, 房屋毁坏越大, 二者的交互项作为 调节项放入，负相关。地震的位置与县城中心越近，损 失也越大，表明房屋密集度越大，损失也会越大。年份 显著正相关与房屋毁坏；对于房屋破坏严重（Serious）

\begin{tabular}{|c|c|c|c|}
\hline Covariates & Est & $\mathbf{S E}$ & P-value \\
\hline \multicolumn{4}{|c|}{ Destructive } \\
\hline Intercept & $-19.0922 * * *$ & 0.0425 & 0.0000 \\
\hline Year & $0.4431 * * *$ & 0.0397 & 0.0000 \\
\hline $\cos ($ month $)$ & 0.1551 & 0.2691 & 0.5644 \\
\hline $\sin ($ month) & -0.0326 & 0.3225 & 0.9196 \\
\hline Magnitude & $1.9257 * * *$ & 0.1262 & 0.0000 \\
\hline Depth & -0.0213 & 0.0422 & 0.6140 \\
\hline Distance & $-1.3004 * * *$ & 0.2505 & 0.0000 \\
\hline Aftershocks & $0.3202 * * *$ & 0.0847 & 0.0002 \\
\hline Magnitude*Aftershocks & $-0.0451 * * *$ & 0.0121 & 0.0002 \\
\hline \multicolumn{4}{|c|}{ Serious } \\
\hline Intercept & $-11.5735^{* * *}$ & 0.0439 & 0.0000 \\
\hline Year & 0.0350 & 0.0341 & 0.3057 \\
\hline $\cos ($ month $)$ & 0.2152 & 0.2529 & 0.3948 \\
\hline $\sin ($ month) & -0.2831 & 0.2853 & 0.3211 \\
\hline Magnitude & $1.5781 * * *$ & 0.1025 & 0.0000 \\
\hline Depth & 0.0193 & 0.0374 & 0.6064 \\
\hline Distance & $-1.6884 * * *$ & 0.3087 & 0.0000 \\
\hline Aftershocks & 0.1248 & 0.0873 & 0.1529 \\
\hline Magnitude*Aftershocks & -0.0175 & 0.0125 & 0.1611 \\
\hline \multicolumn{4}{|c|}{ Medium } \\
\hline Intercept & $-8.6836^{* * * *}$ & 0.0436 & 0.0000 \\
\hline Year & $0.1518 * * *$ & 0.0282 & 0.0000 \\
\hline $\cos ($ month $)$ & -0.0854 & 0.2061 & 0.6785 \\
\hline $\sin ($ month $)$ & $-0.6088 * * *$ & 0.2275 & 0.0075 \\
\hline Magnitude & $1.0600 * * *$ & 0.0884 & 0.0000 \\
\hline Depth & 0.0771 & 0.0327 & 0.1183 \\
\hline Distance & $-0.8607 * * *$ & 0.2809 & 0.0022 \\
\hline Aftershocks & $0.1516^{*}$ & 0.0874 & 0.0830 \\
\hline Magnitude*Aftershocks & $-0.0212 *$ & 0.0125 & 0.0895 \\
\hline \multicolumn{4}{|c|}{ Minor } \\
\hline Intercept & $-6.7721 * * *$ & 0.0346 & 0.0000 \\
\hline Year & 0.01630 & 0.024 & 0.4976 \\
\hline $\cos ($ month $)$ & 0.24140 & 0.1754 & 0.1688 \\
\hline $\sin ($ month $)$ & $-0.7147 * * *$ & 0.1933 & 0.0002 \\
\hline Magnitude & $1.1560 * * *$ & 0.0753 & 0.0000 \\
\hline Depth & 0.0396 & 0.0279 & 0.1548 \\
\hline Distance & $-0.9942 * * *$ & 0.2261 & 0.0000 \\
\hline Aftershocks & 0.0264 & 0.0746 & 0.7233 \\
\hline Magnitude*Aftershocks & -0.0036 & 0.0107 & 0.7389 \\
\hline$\sigma^{2}$ & 0.6171 & & \\
\hline$\tau_{1}^{2}$ & 0.0110 & & \\
\hline$\tau_{2}^{2}$ & 0.0233 & & \\
\hline$\tau_{3}^{2}$ & 0.2958 & & \\
\hline$\tau_{4}^{2}$ & 0.1325 & & \\
\hline
\end{tabular}

表 1: 实证结果

EST.参数估计值; SE.标准误; ${ }^{*} \mathrm{P}<0.1$, ** $\mathrm{P}<0.05,{ }^{*} * * \mathrm{P}<0.01$.

主要还是表现在地震的首次震级与发震位置, 震级越大 严重破坏程度越大，同时与县城中心距离越小，损失越 大；对于房屋破坏中等 (Medium) 表现出年份和月份的 叠加效应, 震级越大损失越大, 地理位置也和严重破坏 程度保持高度一致性, 距离越远损失越小; 对于房屋破 
坏轻微（Minor）月份效应明显, 震级和地理位置表现 出显著性, 震级越大, 损失越大; 距离县城越近, 损失 越大。

结合地震震级, 地震位置, 发震年月, 地震深度因 素计算出为 0.6171 , 不同类别差异的估计数房屋毁坏

（Destructive），房屋破坏严重（Serious），房屋破坏 中等 (Medium) , 房屋破坏轻微 (Minor) 的为 0.0110 , 为 0.0233 , 为 0.2958 , 为 0.1325 , 估计的不同 类别差异之间的明显差异支持了联合混合模型。

\section{III. 结论与建议}

科学及时的评估地震自然灾害造成的房屋损失能够 有助于政府在第一时间进行人员救助、物资发放, 减少 灾害损失。现有研究很少考虑房屋损失程度的差异性, 本文首次将联合混合模型运用于地震自然灾害的房屋损 失差异性进行实证。实证的结果表明: 一是地震的震级 是房屋损失最为关键的影响因子。造成房屋毁坏

(Destructive）与地震的余震有很大关系, 为此余震的 引入成为后期地震研究的关心变量; 二是引入地震位 置, 采取与县城中心的距离作为变量, 运算发现房屋的 位置越接近县域, 房屋的损失越大, 这说明房屋密集度 越大，造成的损失也就越大; 三是从不同类别差异的估 计的 $\tau_{j}^{2}, j=1,2,3,4$, 估计的不同类别差异之间的明显 差异支持了联合混合模型。

当然, 地震自然灾害的突发性和破坏性, 对地区经 济影响与对建筑物设施的破坏的影响一样值得关注, 后 期也将结合云南、中国、全球地震自然灾害进行拓展研 究。最后, 地震自然灾害造成的灾害损失差异性较大,
为提高模型预测的可信度和精确度, 影响因子需更全面 的收集, 统计部门需要做好全面的数据记录以及灾害因 子多方位的收集。

\section{致谢}

本项目受中国博士后科学基金面上项目 （2018M633640XB）的资助。

\section{参考文献}

[1] http: //teles.ncree.org.tw/default.aspx

[2] Allen T I, David J. Wald, Paul S. Earle, et al. "An Atlas of ShakeMaps and population exposure catalog for earthquake loss modeling," Bulletin of Earthquake Engineering, vol 7, issue 3, pp.701-718, 2009.

[3] Stojanovski P, Dong W. "Simulation model for earthquake casualty estimation," Proceedings of 5USNCEE, Chicago, IL,1994, Paper no.00592.

[4] 帅向华, 杨桂岭, 姜立新. “日本防灾减灾与地震应急工作现状,”. 地 震, vol 24, issue 3, pp. 101-106,2004.

[5] 王瑛, 史培军,王静爱. “云南省农村乡镇地震灾害房屋损失评估,”地 震学报, vol 5, pp. 551-560+582, 2005 .

[6] 孙柏涛, 胡少卿, 王东明. “云南省乡镇农村地震灾害直接经济损失 研究,”地震工程与工程振动, vol 1, pp. 153-158, 2005.

[7] 牛方曲, 高晓路, 季玨. “区域中长期房屋震灾损失评估系统, ”资源科 学, vol 34, issue 2, pp.359-366, 2012.

[8] JinChen, WenLi, Wenkai Chen, Suping Zhang. "Assessment of Earthquake Prevention and Disaster Reduction Capability of CountyLevel Administrative Units in Gansu Province," The Journal of Risk Analysis and Crisis Response, vol 7, issue 4, 2017.

[9] Yan, G., Hasan, M. T., and Ma, R. "Modeling proportions and marginal counts simultaneously for clustered multinomial data with random cluster sizes,"Journal of Applied Statistics, vol 43, issue 6, pp. $1074-1087$ 